Bangladesh Rice J. 22 (1) : 45-56, 2018

\title{
Agro-morphological Characterization and Genetic Diversity of Similar Named Aromatic Rice (Oryza sativa L.) Landraces of Bangladesh
}

\author{
M Z Islam*, N Akter, T Chakrabarty, A Bhuiya, M A Siddique \\ and M Khalequzzaman
}

\begin{abstract}
Thirty-six similar named aromatic rice landraces of Bangladesh were studied to assess the genetic variation for the agro-morphological traits through Mahalanobis $\mathrm{D}^{2}$ statistics. The landraces were grouped into four clusters, where no duplicate landrace was found. The inter-cluster distances were higher than intra-cluster distances indicating wider genetic diversity among the landraces of different clusters. The intra-cluster distances were lower in all the cases reflecting homogeneity of the landraces within the clusters. The principal component analysis showed that the first five components with vector values $>1$ contributed $76.51 \%$ of the total variations. The highest number (13) of landraces was constellated in cluster III and the lowest (3) in cluster I. The intra- and intercluster distances were the maximum in cluster I (0.63) and between clusters I and IV (17.13), respectively and the minimum in cluster II $(0.03)$ and between clusters III and IV (3.86), respectively. The maximum value of inter cluster distance indicated that the landraces belonging to cluster IV were far diverged from those of cluster I. So, it is expected in our results that parent's selection for hybridization from the clusters IV and I may give the desirable heterosis for heterotic rice hybrids. Besides, the cluster mean revealed that the crosses between the genotypes of cluster I with those of clusters IV would exhibit high heterosis for maximum good characters. Hence, yield, grain breadth, days to maturity, culm diameter, ligule length had maximum contribution towards genetic divergence. Besides, evaluation of agro-morphological characters demonstrated that the aromatic rice landraces under the present study possessed a considerable genetic diversity. Therefore, similar and duplicate named aromatic rice landraces need to be conserved in Genebank for future breeding programme.
\end{abstract}

Keywords: Genetic diversity, aromatic rice landraces, agro-morphological characters

\section{INTRODUCTION}

Aromatic rice is a special class of rice with high market value due to its superior grain qualities and pleasant aroma (Singh et al., $2000 a, b)$. The centre of diversity of aromatic rice in India is the foothills of Himalayas, covering Uttar Pradesh, Bihar and the Tarai region of Nepal. From there, aromatic rice germplasm spread to the other states of India and neighbouring countries like Bangladesh and adapted to the local environments (Khush, 2000). Bangladesh has a stock of more than 8,000 rice germplasm of which nearly 100 are aromatic (Hamid et al., 1982; Islam et al., 2016). The farmers of this country still grow their heirloom landraces which not only suit to their flavour but also provide food safety. Bangladesh is also the home to many locally adapted aromatic and quality rice landraces. Despite their low-yield potential, these landraces are grown for their high market and social values.

Aromatic rice landraces native to Bangladesh generally have short bold and medium bold grain type with mild to strong

Genetic Resources and Seed Division, Bangladesh Rice Research Institute, Gazipur 1701, Bangladesh. *Corresponding author's E-mail: zahid.grs@gmail.com 
aroma (Shahidullah et al., 2009; Islam et al., 2013). A considerable number of aromatic rice landraces, namely Chinigura, Kalijira, Kataribhog, Begunbichi, Sakkorkhora and Sakkorkhana are grown in the different districts of Bangladesh. Among the different aromatic rice varieties, Chinigura is the predominant one that covers more than $70 \%$ of rice farms in the northern districts of Naogaon and Dinajpur. Other important aromatic rice varieties are Kalijira (predominantly grown in Mymensingh) and Kataribhog (mainly cultivated in Dinajpur) (Baqui et al., 1997). Most of the aromatic rice varieties in Bangladesh are locally adapted, photoperiod-sensitive and grown during Aman season under rainfed lowland ecosystem. However, the average yield of high yielding rainfed lowland rice is $3.4 \mathrm{t} / \mathrm{ha}$, whereas that of aromatic rice is $2.0-2.3 \mathrm{t} / \mathrm{ha}$ (Das et al., 2005).

Exploring diversity in the landrace collection is very important for identifying new genes and further improvement of the germplasm (Thomson et al., 2007). However, it was identified that duplicate(s) named rice germplasm were cultivated all over Bangladesh (Hamid et al., 1982). As a result, a particular cultivar got many slightly deviated names or even different cultivars got the same name given by different farmers. Hence, similar and duplicate named aromatic rice germplasm need to be studied whether they are same or different. It is true that some small and medium-grained aromatic rice landraces possess excellent aroma and other quality traits like elongation after cooking, taste etc. These could be excellent sources for improving quality in high yielding varieties. The improvement of aromatic rice landraces requires collection and evaluation of existing cultivars of Bangladesh. Genetic study of local germplasm of aromatic rice landraces is the pre-requisite for the development of high yielding varieties. Many researchers have performed studies on genetic diversity with local and high yielding varieties of rice (Siddique et al., 2013; Islam et al., 2014; Akter et al., 2016; Ahmed et al., 2016 and Akter et al., 2018). But such type of studies on similar named aromatic rice landraces has not yet been done. Therefore, the present study was undertaken to assess the genetic variation in 36 similar named aromatic rice landraces of Bangladesh by studying the agromorphological traits.

\section{MATERIALS AND METHODS}

\section{Experimental site}

The experiment was conducted at the research farm of Bangladesh Rice Research Institute (BRRI), Gazipur, during July to December (T. Aman season), 2015. Geographically, the place is located at about $24.00{ }^{\circ} \mathrm{N}$ latitude and 90.25 ${ }^{\circ} \mathrm{E}$ longitude with an elevation of 8.4 meters from the sea level and is characterized by subtropical climate. The soil of the experimental site was clay loam in texture.

\section{Crop materials}

A total of 36 landraces of similar named Aromatic rice germplasm (Table 1) of Bangladesh were selected and collected from Rice Genebank of BRRI, Gazipur. Pregerminated seeds were sown in the seed bed.

\section{Experimental design and setting of the experiment}

The experiment was conducted following randomized complete block design (RCBD) with three replications. Thirty days old seedlings from each entry were transplanted on the $20^{\text {th }}$ August, 2015 using single seedling per hill in $2.4 \mathrm{~m}^{2}$ plot following $25 \mathrm{~cm}$ and 20 $\mathrm{cm}$ space between rows and plants, respectively.

\section{Intercultural operations}

Fertilizers were applied @ 60:20:40:10 kg N, P, $\mathrm{K}$ and $\mathrm{S}$ per hectare. Total quantity of TSP, MP 
Table 1. Information on place of collection, source and local name of the aromatic rice landraces.

\begin{tabular}{|c|c|c|c|c|c|c|c|c|}
\hline $\begin{array}{l}\text { Sl. } \\
\text { no. }\end{array}$ & Landrace & $\begin{array}{l}\text { Code } \\
\text { name }\end{array}$ & $\begin{array}{l}\text { Acc. } \\
\text { no. }\end{array}$ & Season & Upazila/Institute & District & Origin & $\begin{array}{c}1.7 \% \mathrm{KOH} \\
\text { (aroma) }\end{array}$ \\
\hline 1 & Chinigura & $\mathrm{C} 1$ & 6719 & T. Aman & Gazipur Sadar & Gazipur & Bangladesh & $S$ \\
\hline 2 & Chinigura & $\mathrm{C} 2$ & 2412 & T. Aman & - & Dhaka & Bangladesh & LS \\
\hline 3 & Chinigura & $\mathrm{C} 3$ & 4867 & T. Aman & Phulpur & $\begin{array}{l}\text { Mymensing } \\
\mathrm{h}\end{array}$ & Bangladesh & LS \\
\hline 4 & Chinigura & $\mathrm{C} 4$ & 7572 & T. Aman & Habiganj Sadar & Habiganj & Bangladesh & $S$ \\
\hline 5 & Chiniguri & $\mathrm{C} 5$ & 1424 & T. Aman & - & Dhaka & Bangladesh & $S$ \\
\hline 6 & Chiniguri & $\mathrm{C} 6$ & 1880 & T. Aman & Kishoreganj Sadar & Kishoreganj & Bangladesh & $S$ \\
\hline 7 & Sakkorkhora & S1 & 1605 & T. Aman & Bauphal & Patuakhali & Bangladesh & $S$ \\
\hline 8 & Sakkorkhana & S2 & 4761 & T. Aman & Betago & Barguna & Bangladesh & $S$ \\
\hline 9 & Sakkorkhana & S3 & 5338 & T.Aman & Morrlganj & Bagerhat & Bangladesh & $S$ \\
\hline 10 & Sakkorkhana & S4 & 7316 & T.Aman & Nalchity & Jhalakati & Bangladesh & LS \\
\hline 11 & Sakkorkhana & S5 & 7500 & T. Aman & Pirojpur Sadar & Pirojpur & Bangladesh & LS \\
\hline 12 & Sakkorkhora & S6 & 7506 & T. Aman & Pirojpur Sadar & Pirojpur & Bangladesh & $S$ \\
\hline 13 & Kataribhog & K1 & 232 & T. Aman & Trishal & Mymensingh & Bangladesh & LS \\
\hline 14 & Kataribhog & $\mathrm{K} 2$ & 1091 & T.Aman & - & Jessore & Bangladesh & $S$ \\
\hline 15 & Kataribhog & K3 & 1491 & T. Aman & Basail & Tangail & Bangladesh & $S$ \\
\hline 16 & Kataribhog TAPL-78 & K4 & 2505 & T. Aman & BRRI, GRSD & Gazipur & Bangladesh & $S$ \\
\hline 17 & Kataribhog TAPL-79 & K5 & 2506 & T. Aman & BRRI, GRSD & Gazipur & Bangladesh & $S$ \\
\hline 18 & Kataribhog TAPL-80 & K6 & 2507 & T. Aman & BRRI, GRSD & Gazipur & Bangladesh & S \\
\hline 19 & Kataribhog TAPL-81 & K7 & 2508 & T. Aman & BRRI, GRSD & Gazipur & Bangladesh & $S$ \\
\hline 20 & Kataribhog TAPL-82 & K8 & 2509 & T. Aman & BRRI, GRSD & Gazipur & Bangladesh & $S$ \\
\hline 21 & Kataribhog TAPL-83 & K9 & 2510 & T. Aman & BRRI, GRSD & Gazipur & Bangladesh & $S$ \\
\hline 22 & Kataribhog TAPL-84 & K10 & 2511 & T. Aman & BRRI, GRSD & Gazipur & Bangladesh & $S$ \\
\hline 23 & Kataribhog TAPL-85 & K11 & 2512 & T. Aman & BRRI, GRSD & Gazipur & Bangladesh & $S$ \\
\hline 24 & Kataribhog TAPL-86 & K12 & 2513 & T. Aman & BRRI, GRSD & Gazipur & Bangladesh & LS \\
\hline 25 & Kataribhog TAPL-87 & K13 & 2514 & T. Aman & BRRI, GRSD & Gazipur & Bangladesh & LS \\
\hline 26 & Kataribhog TAPL-88 & K14 & 2515 & T. Aman & BRRI, GRSD & Gazipur & Bangladesh & $S$ \\
\hline 27 & Kataribhog & K15 & 4362 & T. Aman & Gazipur Sadar & Gazipur & Bangladesh & $S$ \\
\hline 28 & Kataribhog & K16 & 4363 & T. Aman & Gazipur Sadar & Gazipur & Bangladesh & $S$ \\
\hline 29 & Kataribhog & K17 & 4791 & T. Aman & Kaharole & Dinajpur & Bangladesh & LS \\
\hline 30 & Kataribhog & K18 & 7082 & T. Aman & - & Sylhet & Bangladesh & LS \\
\hline 31 & Begunbichi & B1 & 508 & T. Aman & - & Rangpur & Bangladesh & LS \\
\hline 32 & Begunbichi & B2 & 740 & T. Aman & Rangamati Sadar & Rangamati & Bangladesh & LS \\
\hline 33 & Begunbichi & B3 & 986 & T. Aman & - & Khulna & Bangladesh & $S$ \\
\hline 34 & Begunbichi & B4 & 1465 & T. Aman & Nawabganj & Dhaka & Bangladesh & LS \\
\hline 35 & Begunbichi & B5 & 1678 & T. Aman & - & Faridpur & Bangladesh & $S$ \\
\hline 36 & Begunbichi & B6 & 4088 & T. Aman & Saidpur & Nilphamari & Bangladesh & NS \\
\hline
\end{tabular}

NS: Non-scented, LS: Light scented and S: Scented and TAPL: Transplant Aman Pure Line.

and gypsum were applied at the final land preparation. Urea was applied in three splits at 15,30 and 45 days after transplanting (DAT). Intercultural operations and pest control measures were done as and when necessary.

\section{Agro-morphological trait observation}

Observed variables included both qualitative and quantitative agro-morphological characters of leaf, culm, panicle and grain. Observed qualitative characters included 25 characters namely blade pubescence, blade colour, leaf sheath: anthocyanin colour, basal leaf sheath colour, flag leaf angle, ligule colour, ligule shape, coller colour, auricle colour, culm:anthocyanin colouration of nodes, culm angle, internode colour, culm strength, panicle type, panicle exertion, spikelet: awns in the spiklet, spikelet: length of the longest awn, distribution of awing, awn colour, apiculus colour, stigma colour, lemma and palea colour, lemma and palea pubescence, seed coat (bran) colour, leaf senescence, decorticated grain: scent (aroma) while the observed quantitative 
characters included 14 traits and ten plants from each entry were randomly selected for recording data on ligule length $(\mathrm{mm})$, flag leaf area $\left(\mathrm{cm}^{2}\right)$, culm diameter $(\mathrm{mm})$, effective tiller number, plant height $(\mathrm{cm})$, days to flowering, days to maturity, panicle length $(\mathrm{cm})$, filled grains per panicle, grain length $(\mathrm{mm})$, grain breadth $(\mathrm{mm}), 1000$-grain weight $(\mathrm{g})$ and grain yield per hill $(\mathrm{g})$. The observed qualitative traits were scored based on 'Germplasm Descriptors and Evaluation Form' issued by BRRI (2018) (Table 2).

\section{Aroma test}

Aroma was detected by sniffing and was scored as nonscented, lightly scented and scented following $1.7 \% \mathrm{KOH}$ based method (Sood and E A Siddiq, 1978).

\section{Statistical analysis}

Multivariate analyses were performed in computer using GENSTAT 5.5 programme.

\section{RESULTS AND DISCUSSION}

\section{Qualitative traits characterization}

The present study was aimed at identifying distinct qualitative traits variation among the tested aromatic rice landraces. Polymorphism was found in 17 of the 25 qualitative traits under studied; the non-polymorphic traits were the leaf sheath: anthocyanin colour, basal leaf sheath colour, ligule colour, ligule shape, collar colour, auricle colour, culm anthocyanin colour and stigma colour (Table 2).

Table 2. Classification of aromatic rice landraces based on 25 qualitative characters.

\begin{tabular}{|c|c|c|c|c|c|}
\hline $\begin{array}{l}\text { Sl. } \\
\text { no. }\end{array}$ & $\begin{array}{l}\text { Qualitative } \\
\text { character }\end{array}$ & Classification & Frequency & Landrace (Serial number as in Table 1) & $\begin{array}{c}\text { Frequency } \\
\%\end{array}$ \\
\hline \multirow[t]{2}{*}{1} & Blade pubescence & 2. Intermediate & 11 & $1,7,21,22,23,24,25,26,27,35,36$ & 30.55 \\
\hline & & 3. Pubescent & 25 & $\begin{array}{l}2,3,4,5,6,8,9,10,11,12,13,14,15,16,17,18,19,20,28 \\
29,30,31,32,33,34\end{array}$ & 69.45 \\
\hline \multirow[t]{2}{*}{2} & Blade colour & 1. Pale green & 03 & 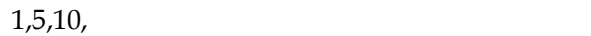 & 8.33 \\
\hline & & 2. Green & 33 & $\begin{array}{l}2,3,4,6,7,8,9,11,12,13,14,15,16,17,18,19,20,21,22 \\
23,24,25,26,27,28,29,30,31,32,33,34,35,36\end{array}$ & 91.67 \\
\hline 3 & $\begin{array}{l}\text { Leaf sheath: } \\
\text { anthocyanin colour }\end{array}$ & 1. Absent & 36 & $\begin{array}{l}1,2,3,4,5,6,7,8,9,10,11,12,13,14,15,16,17,18,19,20 \\
21,22,23,24,25,26,27,28,29,30,31,32,33,34,35,36\end{array}$ & 100 \\
\hline 4 & $\begin{array}{l}\text { Basal leaf sheath } \\
\text { color }\end{array}$ & 1. Green & 36 & $\begin{array}{l}1,2,3,4,5,6,7,8,9,12,13,14,15,16,17,18,19,20,21,22, \\
23,24,25,26,27,28,29,30,31,32,33,34,35,36\end{array}$ & 100 \\
\hline \multirow[t]{2}{*}{5} & Flag leaf angle & $\begin{array}{l}\text { 3. Semi erect }(<30- \\
\left.45^{\circ}\right)\end{array}$ & 18 & $\begin{array}{l}3,4,5,6,7,8,9,10,11,12,13,14,16,17,18,20,21 \\
23,24,25,27,28,29,30,31,32,33,36\end{array}$ & 72.22 \\
\hline & & $\begin{array}{l}\text { 5. Horizaontal } \\
\left(<46-90^{\circ}\right)\end{array}$ & 10 & $1,2,10,11,15,19,22,26,34,35$ & 27.78 \\
\hline 6 & Ligule colour & 1. White & 36 & $\begin{array}{l}1,2,3,4,5,6,7,8,9,10,11,12,13,14,15,16,17,18,19,20 \\
21,22,23,24,25,26,27,28,29,30,31,32,33,34,35,36\end{array}$ & 100 \\
\hline 7 & Ligule shape & 2. 2-cleft & 36 & $\begin{array}{l}1,2,3,4,5,6,7,8,9,10,11,12,13,14,15,16,17,18,19,20 \\
21,22,23,24,25,26,27,28,29,30,31,32,33,34,35,36\end{array}$ & 100 \\
\hline 8 & Collar colour & 1. Pale green & 36 & $\begin{array}{l}1,2,3,4,5,6,7,8,9,10,11,12,13,14,15,16,17,18,19,20 \\
21,22,23,24,25,26,27,28,29,30,31,32,33,34,35,36\end{array}$ & 100 \\
\hline 9 & Auricle colour & 1. Pale green & 36 & $\begin{array}{l}1,2,3,4,5,6,7,8,9,10,11,12,13,14,15,16,17,18,19,20 \\
21,22,23,24,25,26,27,28,29,30,31,32,33,34,35,36\end{array}$ & 100 \\
\hline 10 & $\begin{array}{l}\text { Culm anthocyanin } \\
\text { colour }\end{array}$ & 1. Absent & 36 & $\begin{array}{l}1,2,3,4,5,6,7,8,9,10,11,12,13,14,15,16,17,18,19,2 \\
0,21,22,23,24,25,26,27,28,29,30,31,32,33,34,35 \\
36\end{array}$ & 100 \\
\hline \multirow[t]{3}{*}{11} & Culm angle & 1. $\operatorname{Erect}\left(<30^{\circ}\right)$ & 13 & $6,8,9,16,18,19,20,26,29,30,31,33,36$ & 36.11 \\
\hline & & 3. Intermediate & 15 & $1,2,4,5,7,10,12,13,14,15,17,21,22,25,28$ & 41.67 \\
\hline & & 5. Open & 08 & $3,11,23,24,27,32,34,35$ & 22.22 \\
\hline \multirow[t]{2}{*}{12} & Internode colour & 1. Green & 27 & $\begin{array}{l}1,2,6,7,8,9,10,11,12,13,14,16,17,18,21,22,23,24,25 \\
, 26,27,28,29,30,32,33,34\end{array}$ & 75.00 \\
\hline & & 2. Light gold & 09 & $3,4,5,19,20,23,31,35,36$ & 25.00 \\
\hline
\end{tabular}


Table 2. Continued.

\begin{tabular}{|c|c|c|c|c|c|}
\hline $\begin{array}{l}\text { Sl. } \\
\text { no. }\end{array}$ & $\begin{array}{l}\text { Qualitative } \\
\text { character }\end{array}$ & Classification & Frequency & Landrace (Serial number as in Table 1) & $\begin{array}{c}\text { Frequency } \\
\%\end{array}$ \\
\hline \multirow[t]{3}{*}{13} & Culm strength & 1. Strong & 04 & $6,8,25,36$ & 11.11 \\
\hline & & $\begin{array}{l}\text { 3. Moderately } \\
\text { strong }\end{array}$ & 29 & $\begin{array}{l}1,2,4,5,7,9,11,12,13,14,15,16,17,18,19,20,21,22,23 \\
, 24,26,27,, 28,29,30,31,32,33,34\end{array}$ & 80.55 \\
\hline & & 5. Intermediate & 03 & $3,10,35$ & 8.34 \\
\hline \multirow[t]{3}{*}{14} & Panicle type & 1. Compact & 02 & 16,25 & 5.56 \\
\hline & & 5. Intermediate & 15 & $1,4,8,9,13,14,17,21,24,28,31,32,33,35,36$ & 41.66 \\
\hline & & 9. Open & 19 & $2,3,5,6,7,10,11,12,15,18,19,20,22,23,26,27,29,30,34$ & 52.78 \\
\hline \multirow[t]{3}{*}{15} & Panicle exsertion & 1. Well exerted & 32 & $\begin{array}{l}1,2,3,4,5,6,7,9,10,11,12,13,14,15,16,17,18,19,20,2 \\
1,22,23,26,27,29,30,31,32,33,34,35,36\end{array}$ & 88.88 \\
\hline & & $\begin{array}{l}\text { 3. Moderately well } \\
\text { exerted }\end{array}$ & 02 & 8,28 & 5.56 \\
\hline & & 5. Just exerted & 02 & 24,25 & 5.56 \\
\hline \multirow[t]{2}{*}{16} & $\begin{array}{l}\text { Spikelet: awns in } \\
\text { the spikelet }\end{array}$ & 1. Absent & 23 & $\begin{array}{l}1,2,3,4,5,6,7,8,9,10,11,12,18,22,23,24,25,31,32,33 \\
34,35,36\end{array}$ & 63.89 \\
\hline & & 9. Present & 13 & $13,14,15,16,17,19,20,21,26,27,28,29,30$ & 36.11 \\
\hline \multirow[t]{2}{*}{17} & Distribution of & 1. Tip only & 09 & $14,16,17,21,26,27,28,29,30$ & 69.23 \\
\hline & awning & 3. Upper half only & 04 & $13,15,19,20$ & 30.76 \\
\hline \multirow[t]{3}{*}{18} & Awn colour & 1. Straw & 10 & $13,14,15,16,17,26,27,28,29,30$ & 76.93 \\
\hline & & 3. Brown & 01 & 21 & 7.69 \\
\hline & & 6. Black & 02 & 19,20 & 15.38 \\
\hline \multirow[t]{5}{*}{19} & Apiculus colour & 2. Straw & 23 & $\begin{array}{l}1,2,3,4,5,11,13,14,15,16,17,18,22,23,24,25,26 \\
27,28,29,30,34,36\end{array}$ & 63.89 \\
\hline & & 3. Brown & 01 & 35 & 2.78 \\
\hline & & 4. Red & 03 & $6,21,33$ & 8.33 \\
\hline & & 5. Red apex & 04 & $7,8,9,12$ & 11.11 \\
\hline & & 6. Purple & 05 & $10,19,20,31,32$ & 13.89 \\
\hline 20 & Stigma colour & 1. White & 36 & $\begin{array}{l}1,2,3,4,5,6,7,8,9,10,11,12,13,14,15,16,17,18,19,20 \\
21,22,23,24,25,26,27,28,29,30,31,32,33,34,35,36\end{array}$ & 100 \\
\hline \multirow[t]{5}{*}{21} & $\begin{array}{l}\text { Lemma and palea } \\
\text { colour }\end{array}$ & 0. Straw & 19 & $\begin{array}{l}2,4,11,13,14,15,16,17,18,21,22,23,25,26,27,28,29 \\
30,35\end{array}$ & 52.78 \\
\hline & & 1. Gold & 5 & $1,3,5,24,34$ & 13.89 \\
\hline & & $\begin{array}{l}\text { 3. Brown furrows } \\
\text { on straw }\end{array}$ & 8 & $7,8,9,10,19,20,31,32$ & 22.22 \\
\hline & & 4. Brown & 3 & $6,33,36$ & 8.33 \\
\hline & & $\begin{array}{l}\text { 5. Reddish to light } \\
\text { purple }\end{array}$ & 1 & 12 & 2.77 \\
\hline \multirow[t]{2}{*}{22} & $\begin{array}{l}\text { Lemma and palea } \\
\text { pubescence }\end{array}$ & $\begin{array}{l}\text { 3. Hairs on upper } \\
\text { portion }\end{array}$ & 04 & $7,8,9,32$ & 11.11 \\
\hline & & 4. Short hairs & 32 & $\begin{array}{l}\text { 1,2,3,4,5,6,10,11,12,13,14,15,16,17,18,19,20,21,22 } \\
\text {,23,24,25,26,27,28,29,30,31,33,34,35,36, }\end{array}$ & 88.89 \\
\hline \multirow[t]{3}{*}{23} & $\begin{array}{l}\text { Seed coat (bran) } \\
\text { colour }\end{array}$ & 1. White & 34 & $\begin{array}{l}1,2,3,4,5,6,7,8,9,10,11,12,13,14,15,16,17,18,19,20 \\
21,22,23,24,25,26,27,28,31,32,33,34,35,36\end{array}$ & 94.44 \\
\hline & & 2. Light brown & 01 & 30 & 2.78 \\
\hline & & 5. Red & 01 & 29 & 2.78 \\
\hline \multirow[t]{2}{*}{24} & Leaf senescence & 5. Intermediate & 15 & $3,7,10,11,13,14,16,18,19,22,23,25,27,29,34$ & 41.67 \\
\hline & & 9. Early and fast & 21 & $\begin{array}{l}1,2,4,5,6,8,9,12,15,17,20,21,24,26,28,30,31,32,33 \\
35,36\end{array}$ & 58.33 \\
\hline \multirow[t]{3}{*}{25} & Decorticated grain: & 0. Non scented & 1 & 36 & 2.78 \\
\hline & Scent (aroma) & 1. Lightly scented & 13 & $2,3,10,11,13,24,25,29,30,31,32,34$ & 36.11 \\
\hline & & 2. Scented & 22 & $\begin{array}{l}1,4,5,6,7,8,9,12,14,15,16,17,18,19,20,21,22,23,26 \\
27,28,33,35\end{array}$ & 61.11 \\
\hline
\end{tabular}


Most of the characterized aromatic rice landraces (69.45\%) exhibited pubescent leaf blade while the rest of the landraces exhibited intermediate $(30.55 \%)$ leaf blade pubescence. We also observed in the present study that about $91.67 \%$ of the evaluated aromatic rice landraces exhibited a blade colour green while the remaining landraces $(8.33 \%)$ exhibited pale green. Data in Table 2 also demonstrate variation in flag leaf angle where the percentage of aromatic rice landraces with semi erect and horizontal flag leaf are $72.22 \%$ and $27.78 \%$, respectively. The qualitative characters showing higher variability were culm angle $(36.11 \%$ erect, $41.67 \%$ intermediate and $22.22 \%$ open), internode colour (75\% green and 25\% light gold), culm strength $(11.11 \%$ strong, $80.55 \%$ moderately strong and $8.34 \%$ intermediate), panicle type $(5.56 \%$ compact, $41.66 \%$ intermediate and $52.78 \%$ open), panicle exsertion $(88.88 \%$ well exserted, 5.56\% moderately well exserted and $5.56 \%$ just exserted), spikelet: awns in the spikelet (63.89\% absent and $36.11 \%$ present), distribution of awning (69.23\% tip only and $30.76 \%$ upper half only), awn colour $(76.93 \%$ straw, $7.69 \%$ brown and $19.20 \%$ black).

In the present study, it was also found that most of the tested landraces possessed straw apiculus colour $(76.93 \%)$, straw lemma and palea colour (52.78\%), short hairs on lemma and palea pubescence $(88.89 \%)$, white seed coat colour $(94.44 \%)$, early and fast type leaf senescence $58.33 \%$ ). The present study exhibited high variability in most of the observed qualitative traits of aromatic rice landraces. Similar types of work was also reported by other authors (Bisne and Sarawgi, 2008; Moukoumbi et al., 2011; Ahmed et al., 2016; Mau et al., 2017 and Islam et al., 2017). The unique and similar type aromatic landraces were found for different qualitative agro-morphological characters (Table 3) which could be used in hybridization programme regarding issues like distinctness of new variety and intellectual property rights. Besides, the grain morphology varied considerably in aromatic landraces collected from BRRI genebank (Fig. 1) with respect to awning, colour of awns, lemma and palea colour, lemma and palea pubescence and varied coloured apiculus.

\section{Quantitative traits characterization}

Table 4 shows eigen values (latent roots) and percentage of total variation accounted for them obtained from principle component analysis. The result exposed that the first five components in the PCA with eigen values $>1$, contributed $76.51 \%$ of the total variations among the genotypes for 14 morphological characters.

Islam et al. (2016) observed that the first nine axes accounted about $90 \%$ of the total variations by PCA in 113 aromatic and fine grain rice landraces. On the other hand, Sohrabi et al. (2012) and Chakravorty et al. (2013) observed the contribution of 76.7 and $75.9 \%$ of the first six and four components, respectively to the total variation in rice. Figure 2 presents a two dimension chart $\left(Z_{1}\right.$ $Z_{2}$ ) of the aromatic rice landraces based on the principal axes I and II. As per the scattered diagram the landraces were apparently distributed into four clusters.

Based on the degree of divergence, 36 similar named aromatic landraces were grouped into four clusters on the basis of cluster analysis (Table 5). Maximum 13 landraces were grouped into the cluster III followed by 11 in cluster IV and 9 in cluster II. The cluster I contained the lowest (3) number of landraces. It also revealed that no duplicate was existed among the landraces for the studied characters. Similarly, Fukuoka et al. (2006), Hossain (2008), Sarhadi et al. (2009) and Nascimento et al. (2011) also found no duplicates from cluster analysis using Mahalanobis $\mathrm{D}^{2}$ statistics in rice. 
Table 3. List of unique and similar type Aromatic landraces for different qualitative traits.

\begin{tabular}{|c|c|c|c|}
\hline Characteristic & Index value & Unique type & Similar type \\
\hline $\begin{array}{l}\text { Basal leaf sheath } \\
\text { colour }\end{array}$ & 1. Green & - & All landraces \\
\hline Flag leaf angle & 5. Horizaontal $\left(<46-90^{\circ}\right)$ & $\begin{array}{l}\text { Chinigura (6719), Chinigura (2412), Sakkorkhana } \\
\text { (7316), Sakkorkhana (7500), Kataribhog (1494), } \\
\text { Kataribhog TAPL-81 (2508), Kataribhog TAPL- } \\
\text { 84(2511), Kataribhog TAPL-88 (2515), Begunbichi } \\
\text { (1465) and Begunbichi (1675) }\end{array}$ & - \\
\hline Ligule colour & 1. White & - & All landraces \\
\hline Ligule shape & 2. 2-cleft & - & All landraces \\
\hline Collar colour & 1. Pale green & - & All landraces \\
\hline Auricle colour & 1. Pale green & - & All landraces \\
\hline $\begin{array}{l}\text { Culm anthocyanin } \\
\text { colour }\end{array}$ & 1. Absent & - & All landraces \\
\hline Culm strength & 1. Strong & $\begin{array}{l}\text { Chiniguri (1880), Sakkorkhana (4761), Kataribhog } \\
\text { TAPL-87 (2514) and Begunbichi (4088) }\end{array}$ & - \\
\hline Panicle type & 1. Compact & $\begin{array}{l}\text { Kataribhog TAPL-78 (2504) and Kataribhog TAPL- } \\
87 \text { (2514) }\end{array}$ & - \\
\hline Panicle exsertion & 5. Just exerted & $\begin{array}{l}\text { Kataribhog TAPL-85 (2512) and Kataribhog TAPL- } \\
86 \text { (2513) }\end{array}$ & - \\
\hline $\begin{array}{l}\text { Spikelet: awns in } \\
\text { the spikelet }\end{array}$ & 9. Present & $\begin{array}{l}\text { Kataribhog (232), Kataribhog (1091), Kataribhog } \\
\text { (1491), Kataribhog TAPL-78 (2505), Kataribhog } \\
\text { TAPL-79 (2506), Kataribhog TAPL-81 (2508), } \\
\text { Kataribhog TAPL-82 (2508), Kataribhog TAPL-83 } \\
\text { (2510), Kataribhog TAPL-88 (2515), Kataribhog } \\
\text { (4362), Kataribhog (4363), Kataribhog (4791) and } \\
\text { Kataribhog (7082) }\end{array}$ & - \\
\hline \multirow[t]{4}{*}{ Apiculus colour } & 3. Brown & Begunbichi (1678) & - \\
\hline & 4. Red & $\begin{array}{l}\text { Chiniguri (1880), Kataribhog TAPL-83 (2510) and } \\
\text { Kataribhog TAPL-88 (2512) }\end{array}$ & - \\
\hline & 5. Red apex & $\begin{array}{l}\text { Sakkorkhora (1605), Sakkorkhana } \\
\text { Sakkorkhana (5338) and Sakkorkhora (7506) }\end{array}$ & - \\
\hline & 6. Purple & $\begin{array}{l}\text { Sakkorkhana (7316), Kataribhog TAPL-81 (2508), } \\
\text { Kataribhog TAPL-84 (2511), Begunbichi (508) and } \\
\text { Begunbichi (740) }\end{array}$ & - \\
\hline Stigma colour & 1. White & - & All landraces \\
\hline \multirow[t]{3}{*}{$\begin{array}{l}\text { Lemma and palea } \\
\text { colour }\end{array}$} & $\begin{array}{l}\text { 3. Brown furrows on } \\
\text { straw }\end{array}$ & 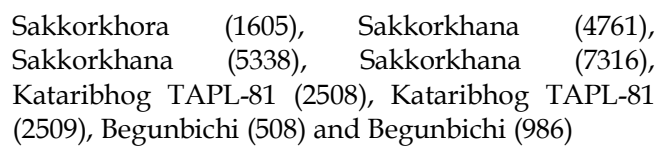 & - \\
\hline & 4. Brown & $\begin{array}{l}\text { Chiniguri (1880), Begunbichi (986) and Begunbichi } \\
(4088)\end{array}$ & - \\
\hline & 5. Reddish to light purple & Sakkorkhora (7506) & \\
\hline $\begin{array}{l}\text { Lemma and palea } \\
\text { pubescence }\end{array}$ & 3. Hairs on upper portion & $\begin{array}{l}\text { Sakkorkhora (1605), Sakkorkhana (4761), } \\
\text { Sakkorkhana (5338) and Begunbichi (740) }\end{array}$ & \\
\hline \multirow{2}{*}{$\begin{array}{l}\text { Seed coat (bran) } \\
\text { colour }\end{array}$} & 2. Light brown & Kataribhog (7082) & - \\
\hline & 5. Red & Kataribhog (4791) & - \\
\hline
\end{tabular}




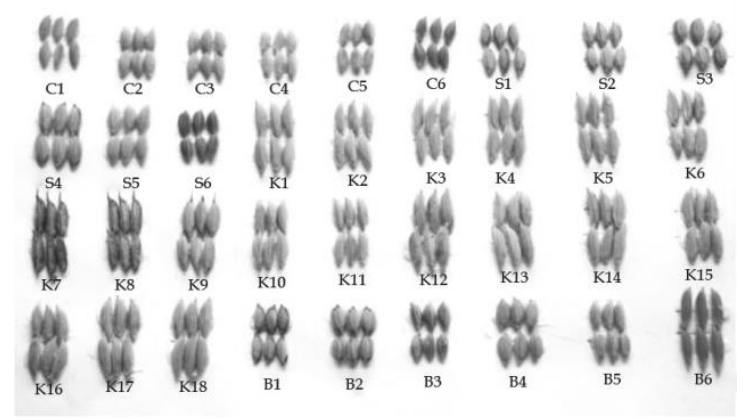

Fig. 1. Variation in grain morphology of 36 similar named aromatic rice landraces.

Note: $\mathrm{C}=$ Chinigura /Chiniguri type, $\mathrm{K}=$ Kataribhog type, $\mathrm{S}=$ Sakkorkhora/Sakkorkhana type and $\mathrm{B}=$ Begunbichi type

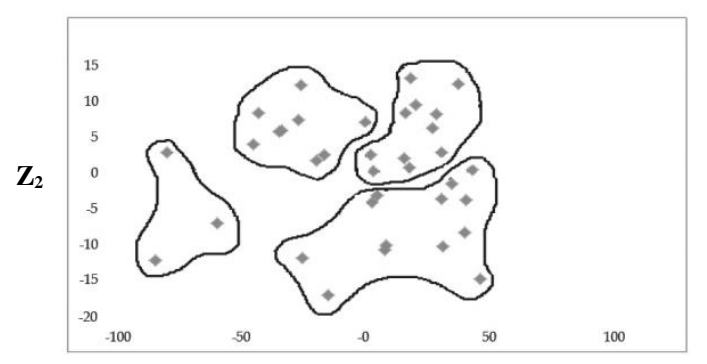

$\mathbf{Z}_{1}$

Fig. 2. Scatter diagram of 36 Aromatic rice landraces based on their principal component scores superimposed with clustering.

Table 4. Latent roots (Eigen values) and their variation in 14 quantitative characters in 36 aromatic landraces.

\begin{tabular}{lcccc}
\hline Character & $\begin{array}{c}\text { Principal } \\
\text { component axes }\end{array}$ & Latent roots & Variation (\%) & $\begin{array}{c}\text { Cumulative \% of } \\
\text { variation }\end{array}$ \\
\hline Ligule length (mm) & PC 1 & 3.551 & 25.37 & 25.37 \\
Flag leaf area $\left(\mathrm{cm}^{2}\right)$ & PC 2 & 2.328 & 16.63 & 42.00 \\
Culm diameter (mm) & PC 3 & 2.126 & 15.19 & 57.19 \\
Effective tiller number & PC 4 & 1.651 & 11.80 & 68.99 \\
Plant height (cm) & PC 5 & 1.053 & 7.52 & 76.51 \\
Days to flowering & PC 6 & 0.798 & 5.71 & 82.22 \\
Days to maturity & PC 7 & 0.747 & 5.34 & 87.56 \\
Panicle length (cm) & PC 8 & 0.579 & 4.14 & 91.7 \\
Filled grains per panicle & PC 9 & 0.451 & 3.22 & 94.92 \\
Grain length (mm) & PC 10 & 0.326 & 2.33 & 97.25 \\
Grain breadth (mm) & PC 11 & 0.260 & 1.86 & 99.11 \\
Grain length-breadth ratio & PC 12 & 0.070 & 0.50 & 99.61 \\
1000 grain weight (g) & PC 13 & 0.053 & 0.37 & 98.00 \\
Yield/hill (g) & PC 14 & 0.002 & 0.02 & 100.00 \\
\hline
\end{tabular}

Table 5. Distribution of 36 aromatic rice landraces into four clusters

\begin{tabular}{|c|c|c|c|}
\hline Cluster & No. of landrace & $\%$ total & Landraces with BRRI accession no. \\
\hline I & 3 & 8.33 & Chinigura(1880), Sakkorkhana (5358), Bengunbichi(508) \\
\hline II & 9 & 25 & $\begin{array}{l}\text { Chinigura (6719), Chinigura (7572), Sakkorkhora (1605), Sakkorkhana (7316), } \\
\text { Kataribhog TAPL-80 (2507), Kataribhog TAPL-84 (2511), Kataribhog (4791), } \\
\text { Bengunbichi (740), Bengunbichi (4088) }\end{array}$ \\
\hline III & 13 & 36.11 & $\begin{array}{l}\text { Chinigura (2412), Chinigura (4867), Chinigura (1424), Sakkorkhana (4761), } \\
\text { Sakkorkhana (7506), Kataribhog (232), Kataribhog (1491), Kataribhog } \\
\text { Kataribhog TAPL-79 (2506), Kataribhog TAPL-85 (2512), KataribhogTAPL-86 } \\
\text { (2513), Kataribhog TAPL-87 (2514), Bengunbichi (986), Bengunbichi (1465) }\end{array}$ \\
\hline IV & 11 & 30.56 & $\begin{array}{l}\text { Sakkorkhana (7500), Kataribhog (1019), Kataribhog TAPL-78 (2505), Kataribhog } \\
\text { TAPL-81 (2508), Kataribhog TAPL-82 (2509), Kataribhog TAPL-83 (2510), } \\
\text { Kataribhog TAPL-88 (2515), Kataribhog (4362), Kataribhog (4363), Kataribhog } \\
\text { (7082), Bengunbichi (4088) }\end{array}$ \\
\hline
\end{tabular}


Table 6 presents Intra and inter-cluster distances. There were marked variations in intra-cluster distances, which ranged from 0.03 to 0.63 . The highest intra-cluster distance was recorded in cluster I (0.63) containing three landraces followed by cluster IV (0.52) with eleven landraces. The lowest intra-cluster distance was observed in cluster II (0.03) having nine number of landraces and the cluster III showed the second lowest intracluster distance $(0.45)$ having the highest (13) number of landraces. Such results indicated that the landraces of cluster I were more heterogeneous as compared to those included in cluster II.

The highest inter-cluster distance was observed between cluster I and IV (17.13) followed by clusters I and III (13.47) (Table 6). The lowest inter-cluster distance was observed between cluster III and IV (3.86) followed by cluster II and III (6.24). The maximum value of inter-cluster distance indicated that the landraces belonging to cluster IV was far diverged from those of cluster I. The minimum inter cluster divergence was observed between cluster III and IV (3.86) indicating that the landraces of these clusters were genetically closed. So, it is expected in our results that parent's selection for hybridization from the clusters IV and I may give the desirable heterosis for heterotic rice hybrids. On the other hand, the inter cluster distances in all the clusters were higher than the intra cluster distances suggesting wider genetic diversity among the landraces of different groups. The results were in agreement with Siddique et al. (2013) and Ahmed et al. (2015). Again, Islam et al. (2016) reported intra and inter-cluster distance ranged from 0.61 to 1.27 and 3.71 to 16.12 , respectively on aromatic and fine rice genotypes.

Table 7 presents the mean performance of 14 characters in four clusters. The highest cluster means for yield, grain breadth, filled grain per panicle, days to maturity and ligule length were obtained from cluster I. The highest panicle length, plant height, effective tiller number, flag leaf area and lowest yield were found in cluster II. On the other hand, the lowest mean value for flag leaf area, days to maturity, filled grains per panicle and highest mean value for 1000 grain weight (TGW) and grain length-breadth ratio were found in cluster IV. The second highest mean yield was found in cluster III. The cluster mean revealed that the crosses between the genotypes of cluster I with those of clusters II and IV would exhibit high heterosis for maximum good characters. Ahmed et al. (2015) and Sohrabi et al. (2012) earlier reported similar trend of conclusions on rice germplasm using $\mathrm{D}^{2}$ statistic

Table 8 presents contributions of the characters towards divergence. The canonical vector analysis revealed that the vectors (vector I and II) were positive for yield, grain breadth, days to flowering, days to maturity, culm diameter, ligule length. Such result indicated that those characters contributed maximum towards divergence. It is interesting that the greater divergence in the present materials due to those characters will offer a good scope for improvement of yield through rational selection of parents for producing heterotic rice hybrids. On the other hand, the other characters namely filled grains per panicle, panicle length, plant height, effective tiller number and leaf area index had the positive value for vector I, similarly, TGW, grain length-breadth ratio and grain length were found positive for vector II, which revealed that those characters was contributed less toward the total genetic divergence. Banumathy et al. (2010) reported that grain yield, days to fifty percent flowering, total grains per panicle and plant height contributed 86.64 per cent towards total divergence. On the contrary, Islam et al. (2014) also reported that panicle number per plant and TGW contributed maximum to the divergence in Sadajira TAPL rice. 
Table 6. Intra (bold) and inter-cluster distances $\left(D^{2}\right)$ for 36 aromatic rice landraces.

\begin{tabular}{ccccc}
\hline Cluster & I & II & III & IV \\
\hline I & $\mathbf{0 . 6 3}$ & 7.73 & 13.47 & 17.13 \\
II & & $\mathbf{0 . 0 3}$ & 6.24 & 10.02 \\
III & & & $\mathbf{0 . 4 5}$ & 3.86 \\
IV & & & & $\mathbf{0 . 5 2}$ \\
\hline
\end{tabular}

Table 7. Cluster means for $\mathbf{1 4}$ quantitative characters in 36 aromatic rice landraces.

\begin{tabular}{lcccc}
\hline Character & I & II & III & IV \\
\cline { 1 - 3 } Ligule length (mm) & 18.24 & 17.11 & 16.60 & 17.53 \\
Flag leaf area (cm $\left.{ }^{2}\right)$ & 23.60 & 26.30 & 21.80 & 19.79 \\
Culm diameter (mm) & 3.39 & 3.79 & 3.72 & 3.50 \\
Effective tiller number & 11.00 & 14.00 & 13.00 & 12.00 \\
Plant height (cm) & 106.83 & 112.65 & 112.22 & 108.84 \\
Days to flowering & 103.00 & 98.00 & 96.00 & 95.00 \\
Days to maturity & 130.00 & 125.00 & 124.00 & 123.00 \\
Panicle length (cm) & 24.40 & 25.47 & 23.40 & 24.55 \\
Filled grains per panicle & 192.00 & 146.00 & 108 & 93.00 \\
Grain length (mm) & 6.23 & 7.09 & 7.49 & 8.30 \\
Grain breadth (mm) & 2.43 & 2.39 & 2.33 & 2.36 \\
Grain length-breadth ratio & 2.59 & 3.01 & 3.25 & 3.57 \\
1000 grain weight (g) & 13.18 & 14.16 & 14.89 & 17.03 \\
Yield/hill (g) & 14.81 & 12.54 & 12.97 & 12.77 \\
\hline
\end{tabular}

Table 8. Latent vectors for 14 characters of 36 Aromatic rice landraces.

\begin{tabular}{lll}
\hline Character & Vector 1 & Vector 2 \\
\hline Ligule length $(\mathrm{mm})$ & 0.1299 & 0.0882 \\
Leaf area index $\left(\mathrm{cm}^{2}\right)$ & 0.4203 & -0.0873 \\
Culm diameter $(\mathrm{mm})$ & 0.2325 & 0.0121 \\
Effective tiller number & 0.1216 & -0.2448 \\
Plant height $(\mathrm{cm})$ & 0.0853 & -0.3006 \\
Days to flowering & 0.3409 & 0.4346 \\
Days to maturity & 0.3136 & 0.4394 \\
Panicle length $(\mathrm{cm})$ & 0.0597 & -0.2584 \\
Filled grains per panicle & 0.4595 & -0.0204 \\
Grain length (mm) & -0.3558 & 0.2748 \\
Grain breadth (mm) & 0.0590 & 0.2161 \\
Grain length-breadth ratio & -0.4270 & 0.2146 \\
1000 grain weight $(\mathrm{g})$ & -0.2786 & 0.4538 \\
Yield/hill $(\mathrm{g})$ & 0.1591 & 0.2358 \\
\hline
\end{tabular}

\section{CONCLUSIONS}

The traditional Aromatic rice landraces can propose a valuable gene pool. They have considerable range of genetic variations for the studied 25 qualitative and 14 quantitative agromorphological characters. The cluster analysis to be found 36 aromatic rice landraces into four groups. The highest inter- cluster distance was observed between clusters I and IV followed by clusters I and III. The maximum value of intercluster distance indicated that the landraces belonging to cluster IV were far diverged from those of cluster I. So, it is expected in our results that parent's selection for hybridization from the clusters IV and I may give the desirable heterosis for heterotic rice hybrids. Besides, the cluster mean revealed that the crosses between the landraces of cluster I with those of cluster IV would exhibit high heterosis for maximum good characters. Moreover, the studied 36 duplicate named aromatic rice landraces are not similar landraces for the studied agro-morphological characters. So, molecular characterizations using simple sequence repeats (SSRs) markers of the studied landraces are required for the confirmation of duplication and also for QTL mapping.

\section{REFERENCES}

Ahmed, M S, S Parveen, M K Bashar and A K M Shamsuddin. 2015. Genetic divergence of balam rice (Oryza sativa L.) germplasm of Bangladesh. Bangladesh Rice J. 19 (1): 9-14.

Ahmed, M S, M K Bashar and A K M Shamsuddin. 2016. Study of qualitative characters of balam rice (Oryza sativa L.) landraces of Bangladesh. Rice Genomics and Genetics. 7 (1):1-8.

Ahmed, M S, M Khalequzzaman, M K Bashar and A K M Shamsuddin. 2016. Agro-morphological, physico-chemical and molecular characterization of rice germplasm with similar names of Bangladesh. Rice Sci. 23 (4): 211-218.

Akter, N, H Begum, M Z Islam, M . Siddique And M Khalequzzaman. 2018. Genetic diversity in Aus rice (Oryza Sativa L.) genotypes of Bangladesh. Bangladesh J. Agril. Res. 43 (2): 253-266. 
Akter, N, M Z Islam, M A Siddique, T Chakrabarty, M Khalequzzaman and M A Z Chowdhury. 2016. Genetic diversity of Boro rice (Oryza sativa L.) landraces in Bangladesh. Bangladesh J. Pl. Breed.Genet. 29 (2): 33-40.

Banumathy, S, R Manimaran, A Sheeba, N Manivannan, B Ramya, D Kumar and G V Ramasubramanian. 2010. Genetic diversity analysis of rice germplasm lines for yield attributing traits. Electronic Journal of Plant Breeding, 1 (4): 500-504.

Baqui, M A, M E Ham, D Jones, and R Straingfellow. 1997. The export potential of traditional varieties of rice from Bangladesh. Bangladesh Rice Research Institute, Gazipur, Bangladesh.

Bisne, R and A K Sarawgi. 2008. Agro-morphological and quality characterization of badshah bhog group from aromatic rice germplasm of Chhattisgarh. Bangladesh J. Agril. Res. 33 (3): 479-492.

BRRI (Bangladesh Rice Research Institute). 2018. Germplasm Descriptors and Evaluation Form. BRRI, Gazipur, Bangladesh.

Chakravorty, A, P D Ghosh and P K Sahu. 2013. Multivariate analysis of phenotypic diversity of landraces of rice of West Bengal. American J. Exp. Agric. 3 (1): 110-23.

Das, T. 2005. Rices in Bangladesh. Dhanmondi, Dhaka1205, Bangladesh, India.

Datt, S, S C Mani and S Datt. 2003. Genetic divergence in elite genotypes of basmati rice (Oryza sativa $\mathrm{L}$ ). Indian J. Genet. Plant Breed. 63 (1): 73-74.

Fukuoka, S, T D Suu, K Ebanna and L N Trinh. 2006. Diversity in phenotypic profiles in landraces populations of Vietnamese rice: a case study of agronomic characters for conserving crop genetic diversity on farm. Genetic Resources and Crop Evol. 53: 753-761.

Hamid, A, N Uddin, M Haque and E Haque. 1982. Deshi Dhaner Jat (Bangla), Publication no.59, Bangladesh Rice Research Institute, Gazipur, Bangladesh.

Hossain, M Z. 2008. Genetic diversity study in fine grain and aromatic landraces of rice (Oryza sativa L.) by morphophysico-chemical characters and micro-satellite DNA markers, PhD Thesis, Department of Genetics and Plant Breeding, BSMRU, Gazipur, Bangladesh.

Islam, M Z, S Banik, M A Haque, M A Siddiquee, M Khalequzzaman and M A K Mian. 2013. Physico-chemical and cooking properties of local aromatic rice germplasm in Bangladesh. Eco-Friendly Agril. J. 6 (10): 243-248.

Islam, M Z, M A Siddique, E S M H Rashid, M S Ahmed and M Khalequzzaman. 2014. Genetic diversity in sadajira rice (Oryza sativa L.) germplasm. The Agriculturists. 12 (1): 26-32.

Islam, M Z, M Khalequzzaman, M K Bashar, N A Ivy, M M Haque and M A K Mian. 2016. Variability Assessment of Aromatic and Fine Rice Germplasm in Bangladesh Based on Quantitative Traits. The Scientific World Journal, Vol. 2016, Article ID 2796720, http://dx.doi.org/10.1155/2016/2796720

Islam, M Z, M Khalequzzaman, M A Siddique, N Akter, M S Ahmed and M A Z Chowdhury. 2017. Phenotypic characterization of Jhum rice (Oryza sativa L.) landraces collected from Rangamati district in Bangladesh. Bangladesh Rice J. 21 (1): 47-57.

Khatun M T, M M Hanafi, M R Yusop, M Y Wong, F M Salleh and J Ferdous. 2015. Genetic variation, heritability, and diversity analysis of upland rice (Oryza sativa L.) genotypes based on quantitative traits. Bio Med Research International, Article ID 290861.

Khush, G S .2000. Taxonomy and origin of rice. In: Singh RK, Singh US, Khush GS (eds) Aromatic rices. Oxford and IBH, New Delhi, pp 5-13.

Mau, Y S, J ER Markus, S S Oematan, A S S Ndiwa, D D Handoko, A Nasution and K Makbul.2017. Genetic diversity of red and black upland rice accessions from East Nusa Tenggara, Indonesia as revealed by agro morphological characters. Biodiversitas. 18 (1): 197-211.

Moukoumbi, Y D, M Sie, R Vodouhe, B N Dri, B Toulou, S A Ogunbayo and A Ahanchede,. 2011. Assessing phenotypic diversity of interspecific rice varieties using agro-morphological characterization. Journal of Plant Breeding and Crop Science. 3 (5): 74-86.

Nascimento, W F, E F Silva and E A Veasey. 2011. Agromorphological characterization of upland rice accessions. Sci. Agric. 68 (6): 652-60.

Roy, S K, A Kundu, S P Chand and B K Senapati. 2004. Diversity of panicle characters in Aman rice (Oryza sativa L). Environ. Ecol. 22 (Spl-3): 500503.

Sarhadi, W A, T Ookawa, T Yoshihashi, A K Madadi, W Yosofzai, Y Oikawa and Y Hirata. 2009. Characterization of aroma and agronomic traits in Afghan native rice cultivars. Plant Production Sci. 12: 63-69.

Shahidullah, S M, M M Hanafi, M. Ashrafuzzaman, M R Ismailand A Khair. 2009. Genetic diversity in grain quality and nutrition of aromatic rices. African J. Biotech., vol. 8, no. 7, pp. 1238-1246.

Siddique, M A, M Khalequzzaman, M Z Islam, M S Ahmed and ESMH Rashid. 2013. Genetic 
diversity in local Boro rice (Oryza Sativa L.) genotypes of Bangladesh. Bangladesh J. Pl. Breed. Genet., 26 (1): 19-24.

Singh, R K, Gautam P L, Saxena S, Singh S. 2000a. Scented rice germplasm: conservation, evaluation and utilization. In: Singh R K, Singh U S, Khush G S (eds) Aromatic rices. Oxford \& IBH, New Delhi, pp 107-133.

Singh, R K, U S Singh, G S Khush, R Rohilla, J P Singh, G Singh and K S Shekhar. 2000b. Small and medium grained aromatic ricesof India. In: Singh RK, Singh US, Khush GS (eds) Aromatic rices. Oxford \& IBH, New Delhi, pp 155-177.
Sohrabi, M, M Y Rafii, M M Hanafi, A S N Akmar and M A Latif. 2012. Genetic diversity of upland rice germplasm in Malaysia based on quantitative traits. The Scientific World Journal, 416291: 1-9.

Sood, B C and E A Siddiq. 1978. A rapid technique for scent determination in rice. Indian Journal of Genetics and Plant Breeding. 38: 268-271.

Thomson, M J, E M Septiningsih, F Suwardjo, T J Santoso, T S Silitonga and S R McCouch. 2007. Genetic diversity analysis of traditional and improved Indonesian rice (Oryza sativa L.) 481 germplasm using microsatellite markers. Theor Appl Genet, 114 (3): 559-568. 